\section{Las trabajadoras de la salud: vida, trabajo y trastornos mentales}

\author{
Female health workers: \\ lifestyle, work, and psychiatric disorders
}

1 Maestría en Ciencias
en Salud en el Trabajo,
Universidad Autónoma
Metropolitana, Unidad
Xochimilco, México DF.
2 Hospital de
Ginecobstetricia No. 4,
Instituto Mexicano del
Seguro Social, México DF.
3 Instituto de Investigaciones
en Matemáticas Aplicadas
y en Sistemas, Universidad
Nacional Autónoma
de México, México DF.
Correspondencia
M. Noriega
Maestría en Ciencias
en Salud en el Trabajo,
Universidad Autónoma
Metropolitana.
Calzada del Hueso 110o, Col.
Villa Quietud, Delegación
Coyacán 04960, México DF.
mnoriega@correo.xoc.uam.mx

Abstract

The relationships were studied between daily life, work, and mental disorders in female health workers from the Mexican Health Insurance Institute. The study sample $(n=170)$ included female physicians, nurses, laboratory workers, and medical assistants. Primary data were obtained through an interview which had been previously validated in a population of workers in Mexico. Relationships were found between mental disorders and all facets of women's lives. In relation to the domestic environment, women with higher rates of mental disorders were those who were mothers, had more children, did not have household help, and had husbands or partners. Prevalence of mental disorders in relation to paid work was associated with the length of the workday, absenteeism, and lack of job content. Skills development, job satisfaction, and creativity had a "protective" or preventive effect against mental disorders and fatigue. The main risks and conditions that functioned as stressors were heat, noise, physical effort, awkward positions, and intense, repetitive work.

Working Conditions; Living Conditions; Mental Disorders; Fatigue
Mariano Noriega 1

Guadalupe Gutiérrez ${ }^{2}$

Ignacio Méndez 3

Margarita Pulido 1

\section{Introducción}

La relación entre la "vida cotidiana" 1 en el ámbito doméstico, el trabajo asalariado y la condición de ser mujer establece complejos problemas para el estudio y la causalidad de las enfermedades. Tradicional y culturalmente en países como México a la mujer se le ha adjudicado un rol en donde la principal ocupación es la de dedicarse a las actividades domésticas. Sin embargo, las nuevas demandas provocadas por la crisis, principalmente para incrementar los ingresos económicos de la familia, han obligado a muchas mujeres a colocarse en algún sitio del aparato productivo. Empero, también ha obligado a tomar la decisión de ausentarse, eventualmente, de las tareas domésticas, lo que ha provocado cambios importantes, no sólo en su propia percepción, sino también en la dinámica familiar.

Se han identificado diferencias significativas entre las mujeres que se dedican únicamente al hogar y aquéllas que combinan estas actividades con el trabajo asalariado. La mujer trabajadora - que tiene un mayor control sobre su trabajo - experimenta mayor bienestar psicológico en comparación con la que tiene bajo control sobre su trabajo o con aquélla que nunca ha tenido un trabajo asalariado. Las primeras presentan menos estrés que los otros dos grupos 2. En un sentido similar, la antigüedad laboral influye en la autoestima de las trabaja- 
doras. Las mujeres con menor antigüedad en el trabajo remunerado reportaron menor autoestima que aquéllas con mayor antigüedad, ya que estas últimas se evalúan positivamente como trabajadoras 3 .

Por otro lado, las demandas sociales para las mujeres son diferentes que para los varones: el papel asignado socialmente a aquéllas se cumple siempre y cuando consigan mantener en armonía las relaciones de pareja, del trabajo profesional y de los colegas. La mujer tiene permiso social de ser ama de casa, por consecuencia no es indispensable que se mantenga empleada. Tal parece que la mujer trabajadora lo que consigue con su empleo es obtener satisfacción personal e incrementar su autoestima. A pesar de que son tanto o más capaces que los hombres para desempeñar cualquier trabajo, la educación de las mujeres ha sido dirigida de tal manera que se sienten obligadas a manejar las demandas de su familia, trabajo y casa al mismo tiempo. Así, las mujeres suelen tener varios papeles: son madres, esposas, hijas y trabajadoras simultáneamente y logran responder a las demandas de cada uno de estos papeles. Las mujeres pueden manejar dichas situaciones porque están adaptadas a esta realidad 4,5, aunque dicha “adaptación” puede significar un alto precio para su salud.

En fechas recientes se han realizado numerosos estudios sobre las condiciones de trabajo y de salud en el llamado sector terciario o de los servicios. Dentro de este grupo se incluyen a las trabajadoras de la salud. El interés principal por estudiar a este personal ha sido su exposición a riesgos biológicos y su posible causa de enfermedad, en particular, el estudio de la hepatitis y del SIDA se ha convertido en un problema justificadamente prioritario. Sin embargo, las situaciones que más transgreden la integridad física y mental de las trabajadoras de la salud son sus condiciones de trabajo (doméstico y remunerado) y las formas como están organizados 6,7. Esta situación determina condiciones altamente estresantes que favorecen la aparición de trastornos psíquicos y psicosomáticos 8,9,10,11,12,13,14.

El trabajo de los servicios de atención a la salud se caracteriza por tener un objeto de trabajo muy peculiar, ya que éste es un sujeto (el paciente). Si bien es cierto que este tipo de trabajo permite, con frecuencia, desarrollar las capacidades mentales y físicas, también es cierto que la actividad que las trabajadoras de la salud efectúan, contiene elementos que en el momento de interactuar, pueden ser potencialmente nocivos o generadores de enfermedad para el conjunto y para cada una de ellas. Den- tro de estos elementos potencialmente nocivos, los que se encuentran con mayor frecuencia en los servicios de salud son las exigencias laborales, o sea, aquellos elementos derivados de las características que adopta tanto el trabajo propiamente dicho como la forma en que está organizado.

Muchas trabajadoras de la salud consideran su actividad como un trabajo de retos y, de alguna manera, sienten que alcanzan una satisfacción con ella. Sin embargo, creen que para enfrentar estos retos es importante que exista un equilibrio entre las demandas ocupacionales y las características personales, para que esto no represente una sobrecarga de trabajo. Cuando la trabajadora se enfrenta y percibe condiciones desfavorables, la consecuencia puede ser el estrés y los trastornos mentales. En este tipo de mujeres, por la manera en que está organizado el trabajo, generalmente existe una sobrecarga cuantitativa. Junto a esto, uno de los factores de estrés que más sobresale es la excesiva responsabilidad, dado que el trabajo se centra precisamente en la seguridad de los seres humanos en los cuales aplica su conocimiento. Otro de los factores de estrés conocidos es la ambigüedad en las actividades que se realizan, es decir, cuando no hay claridad suficiente de cuáles son las tareas que se deben desempeñar. Así, si hay claridad en las responsabilidades y funciones durante el desarrollo del trabajo, el resultado será satisfactorio. Sin embargo, en el caso contrario las consecuencias pueden ser lamentables 15.

Los trastornos psíquicos y psicosomáticos pueden originarse directamente, tanto por las condiciones de trabajo y los factores de estrés laboral, como por las características de la vida cotidiana en el ámbito doméstico. Se ha encontrado que cuando no hay armonía entre ciertas exigencias laborales (posiciones forzadas, alta velocidad y precisión para efectuar la actividad, rigidez del horario, monotonía) y algunas características personales (experiencia, conocimiento de la tarea, personalidad y percepción del trabajador acerca de la carga de la tarea), o sea, entre las demandas laborales y las individuales, se incrementa claramente la posibilidad de potenciar la aparición de algunos desórdenes mentales 16 .

En el personal al cuidado de la salud, el trabajo estresante es más frecuente en las trabajadoras menos cualificadas, mientras que la carga mental se manifiesta más alta en los grupos cualificados. Entre los elementos que generan mayor carga mental y estrés están: insuficiente entrenamiento y exceso de tensión debida a los horarios. La fatiga se encuentra aso- 
ciada, generalmente, con una carga de trabajo excesiva $9,17,18$.

A pesar de que la mente es una entidad abstracta del cerebro que se encarga de efectuar las funciones que no pueden ser medidas mediante pruebas de laboratorio, sin embargo, las funciones mentales (atención, memoria, emociones, sentimientos, contenido del pensamiento, creatividad) sí pueden ser exploradas y valoradas mediante la aplicación de diferentes pruebas psicológicas, según lo requiera el caso. En este sentido se afirma que la "enfermedad mental” es solamente una hipótesis, ya que un individuo mentalmente sano o enfermo es un ser humano que bajo ciertas circunstancias es o no capaz de hallar soluciones a los problemas que enfrenta. De esta manera el comportamiento humano no se determina sólo por la individualidad, sino básicamente en relación con el contexto social. Por lo tanto, los límites de los trastornos psíquicos con respecto a la normalidad son bastante vagos. Sin embargo, en general, cualquier individuo que tenga alguna alteración mental no logra enfrentar de la mejor forma, es decir, con una actitud socialmente aceptada, sus dificultades personales y darles solución. La alteración puede agravarse por los estímulos provenientes de las condiciones de la vida cotidiana. Así, la frustración, el conflicto, las presiones externas pueden potenciar el estrés y los trastornos mentales.

El estrés es la respuesta del organismo frente a diversos mecanismos que producen tensión o agresión. Durante algunas situaciones, el estrés nos impulsa a realizar acciones que nos permiten alcanzar objetivos y metas que benefician nuestra vida personal y colectiva. En cambio en otras situaciones el estrés puede traer consecuencias lamentables. Cuando el individuo percibe, de manera repentina o a través de una presencia constante, condiciones desfavorables este estado de estrés puede manifestarse en síntomas a nivel fisiológico y mental. La American Psychiatric Association 19,20 clasifica los diversos síndromes y trastornos de inadaptación de la personalidad como trastornos mentales. Los trastornos más comunes han sido clasificados de acuerdo a las características y síntomas presentes en los cuadros clínicos. De manera general, aunque un poco arbitraria, los síntomas más comunes de los trastornos mentales se pueden clasificar en: psíquicos o psicológicos (irritabilidad, trastornos del sueño, sensaciones de ansiedad, depresión excesiva, temor, trastornos de la conciencia); psicosomáticos (colitis, gastritis, neurodermatitis, hipertensión, dolor precordial, cefalea tensional, migraña); y de agotamiento (fatiga).
Se ha investigado la frecuencia de síntomas y principales fuentes de estrés ocupacional percibidos por grupos de trabajadores de la salud. Los síntomas predominantes fueron de ansiedad y depresión. Las mayores causas de insatisfacción en más de la mitad de los sujetos son: sentir su trabajo poco reconocido y valorado y la escasa posibilidad de controlarlo. Entre los factores de tensión destaca el sentimiento de tener que responder a muchas personas, el apremio de tiempo y las condiciones de trabajo físico-ambientales inadecuadas 6 . Las diferencias encontradas indican una situación menos favorable para los grupos menos cualificados, lo cual tiende a apoyar las tesis de Karasek \& Theorell 21 en términos del mayor riesgo de estrés y morbilidad, en general, de las personas sometidas a altas demandas, pero con escasa autonomía o control sobre el modo como realizan su trabajo. Así, la baja realización profesional es una variable central para predecir el agotamiento relacionado con el trabajo 22 . La insatisfacción laboral y el elevado estrés fueron los aspectos principales como determinantes de los problemas de salud mental en las trabajadoras de la salud 23 . Asimismo, las altas demandas de trabajo predicen significativamente altos niveles de fatiga y ésta, a su vez, está asociada con altos niveles de enfermedad mental 18. Las trabajadoras de la salud tienen las más altas tasas de admisión hospitalaria por trastornos mentales y tasas de admisión elevadas en centros de salud mental 24 . La prevención y tratamiento incluyen mayor control laboral, reuniones de grupo, mejora en la comunicación hacia arriba y hacia abajo, mayor reconocimiento del mérito individual, rediseño laboral y orientación de los requerimientos del trabajo 25.

\section{Material y métodos}

La población estudiada se conformó con las trabajadoras de una Unidad de Medicina Familiar (UMF), del Instituto Mexicano del Seguro Social (IMSS), ubicada en la zona sureste de la Ciudad de México. La UMF cuenta con un total de 380 trabajadores, de los cuales el 93,0\% (354) se encuentran sindicalizados y el 7,0\% (26) son personal de confianza. Se estudió a la población femenina $(n=217)$, la cual se compone de médicas, enfermeras, personal de laboratorio y asistentes médicas. La no respuesta fue del $22,0 \%$, o sea, 47 trabajadoras. Así, el grupo en estudio quedó conformado con 170. La sustitución no fue posible por haberse aplicado el estudio a toda la población femenina de dicho 
centro laboral. La distribución por edad y antigüedad de la población estudiada en relación con la población que no respondió el cuestionario es muy similar y no se encontraron diferencias significativas.

El diseño del estudio fue transversal. La información se recabó de tres fuentes: la nómina de los trabajadores de la UMF, una guía de observación del proceso de trabajo y una encuesta individual de autocumplimentación, validada en población trabajadora mexicana. Por medio de la nómina del centro de trabajo se recabaron algunas de las variables demográficas fundamentales. Con la guía de observación se recogió información sobre las características del trabajo y de las condiciones ambientales del centro laboral. Con la encuesta se recogieron algunas de las características de la vida cotidiana en el ámbito doméstico; las características básicas del proceso laboral remunerado y la percepción de las trabajadoras sobre el mismo; los factores de estrés laboral a los que se exponen más constantemente y, por último, la sintomatología psíquica, psicosomática y de fatiga.

Las variables independientes que se estudiaron fueron agrupadas en: demográficas (edad, estado civil), vida cotidiana en el ámbito doméstico (presencia de hijos, número y edades de los hijos, ayuda en actividades domésticas), trabajo asalariado (servicio en el que participa, puesto de trabajo, antigüedad en el IMSS, duración de la jornada de trabajo), ausentismo (retardos, licencias, incapacidades), valoración del trabajo (desarrollo de habilidades y destrezas, creatividad e iniciativa, satisfacción, percepción del daño a la salud), causantes de estrés laboral (calor, ruido, esfuerzo físico, posiciones forzadas, trabajo intenso, monotonía, trabajo repetitivo).

La edad y la antigüedad en el centro de trabajo se consideraron como variables confusoras por lo que se controlaron por medio de modelos de regresión logística, dado que las variables dependientes son categóricas.

El método que se siguió para el análisis por conglomerados (clusters) fue el siguiente: se incorporaron todas las variables independientes en estudio, ya fueran numéricas o categóricas. En este último caso las variables se dicotomizaron para poder trabajarlas como numéricas, pues el análisis por conglomerados no admite variables categóricas. Se estudiaron diferentes conglomerados para decidir con cuáles y cuántos trabajar. Se eligió trabajar con dos conglomerados, pues mostraban mejor que ningún otro la clara diferenciación entre las variables en estudio, es decir, eran los que diferenciaban mejor los grupos con mayor y menor grado de exposición. Después se relacionaron los conglomerados con los trastornos mentales, o sea, con las variables dependientes (todas ellas categóricas) y se analizaron una a una para ver si la diferencia entre los conglomerados era significativa.

\section{Resultados}

La UMF estudiada ofrece servicios de atención primaria a la salud. El proceso de atención no siempre sigue una misma dirección, ya que no necesariamente participa secuencialmente cada una de las áreas de servicios. La razón por la que ocurre de esta manera se debe a que cada área de servicio tiene funciones específicas. Las actividades están organizadas en dos turnos: matutino y vespertino, con distintos horarios de entrada y de salida según las necesidades. Los principales servicios son, de manera agrupada, los siguientes: Atención Médica Continua, Materno-Infantil, Medicina Familiar, Servicio Dental, Laboratorio Clínico, Medicina Preventiva, Planificación Familiar y Medicina del Trabajo. Las trabajadoras estudiadas se distribuyeron, por puesto de trabajo, de la siguiente forma: alrededor de una tercera parte $(34,1 \%)$ son asistentes médicas y otra tercera parte $(32,4 \%)$ son enfermeras. Las médicas son el $19,4 \%$ y el $14,1 \%$ son químicas y personal de laboratorio.

De manera general, los factores de estrés laboral a los que se encuentran expuestas las trabajadoras de la UMF son similares por tipo de servicio y por puesto de trabajo. Esto se debe a que son más las características comunes que comparten como mujeres y trabajadoras de la salud que las diferencias específicas por su área o puesto de trabajo. Se encontraron como las principales exigencias, para el conjunto de la población estudiada, las relacionadas con el apremio de tiempo. La tasa general de trastornos psíquicos y psicosomáticos y fatiga para el conjunto de estas trabajadoras fue de 54,0 por 100 , es decir, que de cada dos trabajadoras una tuvo alguno de estos padecimientos. Si se agrupan los diagnósticos en tres conjuntos patológicos: los trastornos psicosomáticos tienen una tasa de 29,0 por cada 100 trabajadoras. Los trastornos psíquicos se presentan en 16,0 de cada 100 y la fatiga en 9,4 .

\section{Vida cotidiana en el ámbito doméstico}

La información muestra cómo algunas situaciones estresantes en el ámbito doméstico pueden tener un impacto importante en la salud men- 
tal de las trabajadoras. Entre las variables que, directa o indirectamente, ilustran más claramente la vida cotidiana en relación con la morbilidad mental están: si la mujer tiene o no pareja; si tiene hijos y el número de ellos; y si cuenta con ayuda en el trabajo doméstico, ya que todos estos elementos tienen relación con la carga de trabajo y con los causantes de estrés o depresores que pueden originar problemas de morbilidad mental.

Los trastornos mentales se elevan en el caso de las trabajadoras que tienen pareja. El riesgo es 50,0\% mayor de padecer algún padecimiento. Además, las trabajadoras que tienen hijos tuvieron un riesgo casi tres veces mayor que las que no tienen hijos. Asimismo, se exploró el número total de hijos. Se agruparon en tres grandes categorías: las que no tenían hijos, las que tenían de uno a dos hijos y las que tenían tres hijos o más. El riesgo se va incremen- tando en relación con el número de hijos (Tabla 1). Es importante mencionar que no se encontraron diferencias significativas entre las madres que tenían hijos menores de cinco años de aquéllas que tenían iguales o mayores a esa edad. Se analizó también a la población estudiada en función de si contaba con alguna ayuda para llevar a cabo las actividades domésticas. En la misma tabla se puede observar que existe un riesgo casi del doble de tener algún trastorno psíquico o psicosomático para aquellas mujeres que no cuentan con ayuda para desarrollar estas actividades.

\section{Condiciones y valoración \\ del trabajo remunerado}

En relación con la extensión de la jornada laboral se encontró que casi dos terceras partes de las trabajadoras $(62,9 \%)$ tienen que laborar

Razón de prevalencia de trastornos mentales en las trabajadoras en relación con sus condiciones de vida, duración de la jornada y ausentismo del trabajo.

\begin{tabular}{|c|c|c|c|c|c|}
\hline \multirow[t]{3}{*}{ Condición } & \multicolumn{5}{|c|}{ Trastornos mentales } \\
\hline & \multicolumn{2}{|c|}{ Sí } & \multicolumn{2}{|c|}{ No } & \multirow[t]{2}{*}{$\mathrm{RP}^{\star}(\mathrm{IC} 95 \%)^{\star \star}$} \\
\hline & $\mathrm{n}$ & $\%$ & $\mathrm{n}$ & $\%$ & \\
\hline \multicolumn{6}{|l|}{ Pareja } \\
\hline $\operatorname{Sin}$ & 20 & 40,8 & 29 & 59,2 & 1,0 \\
\hline Con & 72 & 59,5 & 49 & 40,5 & $1,5(1,04-2,00)$ \\
\hline \multicolumn{6}{|l|}{ Hijos } \\
\hline $\operatorname{Sin}$ & 4 & 21,1 & 15 & 78,9 & 1,0 \\
\hline Con & 88 & 58,3 & 63 & 41,7 & $2,8(1,40-5,30)$ \\
\hline $1-2$ & 53 & 50,0 & 53 & 50,0 & $2,4(1,10-4,90)$ \\
\hline 3 ó más & 24 & 70,6 & 10 & 29,4 & $3,3(1,70-6,70)$ \\
\hline \multicolumn{6}{|l|}{ Ayuda trabajo doméstico } \\
\hline $\operatorname{Sin}$ & 25 & 86,2 & 4 & 13,8 & $1,8(1,30-2,50)$ \\
\hline Con & 67 & 47,5 & 74 & 52,5 & 1,0 \\
\hline \multicolumn{6}{|c|}{ Duración de la jornada asalariada (horas) } \\
\hline 6,5 & 25 & 39,7 & 38 & 60,3 & 1,0 \\
\hline 8,0 & 67 & 62,6 & 40 & 37,4 & $1,6(1,20-2,20)$ \\
\hline \multicolumn{6}{|l|}{ Ausentismo } \\
\hline$<40$ retardos/año & 71 & 50,0 & 71 & 50,0 & 1,0 \\
\hline$\geq 40$ retardos/año & 21 & 75,0 & 7 & 25,0 & $1,5(1,10-2,10)$ \\
\hline Sin licencia & 50 & 48,1 & 54 & 51,9 & 1,0 \\
\hline$\geq 21$ días de licencia & 16 & 94,1 & 1 & 5,9 & $2,0(1,30-2,90)$ \\
\hline Sin incapacidad & 12 & 27,9 & 31 & 72,1 & 1,0 \\
\hline$>40$ días de incapacidad & 36 & 92,3 & 3 & 7,7 & $3,3(2,20-4,90)$ \\
\hline
\end{tabular}

* Razón de prevalencia; ** Intervalo con 95\% de confianza. Modelos de regresión logística ajustados por edad. En todos los casos las diferencias fueron significativas. 
jornadas de ocho horas; el resto labora jornadas de 6,5 horas. Las trabajadoras que laboran jornada completa presentaron mayor morbilidad mental con una razón de prevalencia de 1,6 (IC: 1,2-2,2).

El ausentismo es un fenómeno que se observa cotidianamente en los centros laborales. Las causas son múltiples, entre ellas: las necesidades personales; las mismas condiciones de trabajo y la actitud que la mujer asume con respecto a su trabajo y, una de las más importantes, los problemas de salud. Algunas variables relacionadas con el ausentismo mostraron estrecha relación con los trastornos mentales. La variable más importante, sin duda, fue la de incapacidades médicas, ya que se evidenció una muy estrecha asociación con el conjunto del perfil patológico mental. En menor grado, pero también relevante, fue la asociación entre estos trastornos y los retardos y las licencias en el año (Tabla 1).

El análisis de la información muestra que las incapacidades son un buen indicador en relación con el perfil patológico mental. En la Tabla 2 se aprecia cómo hay un incremento progresivo de la morbilidad mental total, y de cada uno de los conjuntos patológicos, conforme aumentan los días de incapacidad. Se pueden observar morbilidad mental baja en aquellas trabajadoras que no tuvieron incapacidades médicas en el año; morbilidad media en las que tuvieron hasta cuarenta días de incapacidad y morbilidad alta en aquellas mujeres que tuvieron más de cuarenta días de incapacidad. Esto muestra que muchas de las enfermedades de las trabajadoras están relacionadas con trastornos psíquicos y psicosomáticos y con fatiga. Es notorio que el riesgo de fatiga es nueve veces mayor para las trabajadoras que tuvieron más de cuarenta días de incapacidad en el año en relación con las que no tuvieron ni un solo día de incapacidad (Tabla 2).

Para analizar la valoración que la población en estudio tiene sobre su trabajo, se indagó acerca de varios aspectos: el sentimiento de preocupación sobre la posibilidad de que el trabajo le ocasione algún daño a su salud; y, por otro lado, aspectos positivos como: si su trabajo le permitía desarrollar sus habilidades y capacidades; si sentía satisfacción al realizar su actividad; y si su trabajo le permitía desarrollar su creatividad. Las trabajadoras que contestaron que sí estaban preocupadas por los daños a la salud que el trabajo les pudiera ocasionar, tuvieron un riesgo de morbilidad mental mucho más elevado (Tabla 2). El riesgo fue desde 2,0 y hasta 5,5 veces mayor en las trabajadoras con una mayor preocupación por el riesgo a que se someten que aquéllas que no lo consideran así.

En la Tabla 3 podemos apreciar cómo las trabajadoras que contestaron que no sienten bienestar en su trabajo manifestado por: la imposibilidad de desarrollar sus habilidades, por insatisfacción en el trabajo y falta de creatividad, presentan tasas de morbilidad mental mucho más elevadas que aquéllas que sí sienten bienestar.

\section{Causantes de estrés laboral}

Las actuales condiciones de trabajo de la población en estudio determina la presencia de un variado e importante número de riesgos y exi-

Morbilidad mental en relación con incapacidades médicas y con la preocupación de sufrir daños a la salud ocasionados por el trabajo

\begin{tabular}{|c|c|c|c|c|c|c|c|c|c|}
\hline \multirow[t]{3}{*}{ Condición } & \multicolumn{3}{|c|}{ Psicosomáticos } & \multicolumn{3}{|c|}{ Psíquicos } & \multicolumn{3}{|c|}{ Fatiga } \\
\hline & Sí & No & $R P *(I C 95 \%)^{\star \star}$ & Sí & No & $\mathrm{RP}^{\star}(\mathrm{IC} 95 \%)^{\star \star}$ & Sí & No & $\mathrm{RP}^{\star}(\mathrm{IC} 95 \%)^{\star \star}$ \\
\hline & $\%$ & $\%$ & & $\%$ & $\%$ & & $\%$ & $\%$ & \\
\hline \multicolumn{10}{|c|}{ Incapacidad médica } \\
\hline $\operatorname{Sin}$ & 8 & 35 & 1,0 & 3 & 40 & 1,0 & 1 & 42 & 1,0 \\
\hline$\leq 40$ días & 25 & 63 & $1,5(8,00-3,00)^{\star \star \star}$ & 12 & 76 & $2,0(0,60-6,30)^{\star \star \star}$ & 7 & 81 & $3,4(0,50-23,20)^{\star \star \star}$ \\
\hline$>40$ días & 16 & 23 & $2,2(1,10-4,40)$ & 12 & 27 & $4,4(1,50-12,60)$ & 8 & 31 & $8,8(1,70-45,10)$ \\
\hline \multicolumn{10}{|c|}{ Preocupación daños } \\
\hline $\operatorname{Sin}$ & 13 & 62 & 1,0 & 5 & 70 & 1,0 & 2 & 73 & 1,0 \\
\hline Con & 36 & 59 & $2,2(1,30-3,70)$ & 22 & 73 & $3,5(1,50-8,00)$ & 14 & 81 & $5,5(1,60-19,40)$ \\
\hline
\end{tabular}

* Razón de prevalencia; ** Intervalo con 95\% de confianza. Modelos de regresión logística ajustados

por edad y antigüedad en el IMSS; ${ }^{\star \star \star}$ Diferencia no significativa. 
Razón de prevalencia de trastornos mentales en relación con la percepción de las trabajadoras sobre el contenido de su trabajo y con los principales factores de estrés laboral

\begin{tabular}{|c|c|c|c|c|c|}
\hline \multirow[t]{3}{*}{ Condición } & \multicolumn{5}{|c|}{ Trastornos mentales } \\
\hline & \multicolumn{2}{|c|}{ Sí } & \multicolumn{2}{|c|}{ No } & \multirow[t]{2}{*}{$\mathrm{RP}^{*}(\mathrm{IC} 95 \%)^{\star *}$} \\
\hline & $n$ & $\%$ & $\mathrm{n}$ & $\%$ & \\
\hline \multicolumn{6}{|c|}{ Contenido del trabajo } \\
\hline \multicolumn{6}{|c|}{ Desarrollo de habilidades } \\
\hline Sí & 70 & 49,0 & 73 & 51,0 & 1,0 \\
\hline No & 22 & 81,5 & 5 & 18,5 & $1,7(1,20-2,30)$ \\
\hline \multicolumn{6}{|l|}{ Satisfacción } \\
\hline Sí & 77 & 49,4 & 77 & 50,6 & 1,0 \\
\hline No & 15 & 93,8 & 1 & 6,2 & $1,9(1,30-2,70)$ \\
\hline \multicolumn{6}{|l|}{ Creatividad } \\
\hline Sí & 56 & 43,8 & 72 & 56,2 & 1,0 \\
\hline No & 36 & 85,7 & 6 & 14,3 & $2,0(1,50-2,60)$ \\
\hline \multicolumn{6}{|c|}{ Factores de estrés } \\
\hline \multicolumn{6}{|c|}{ Calor } \\
\hline No & 39 & 43,3 & 51 & 56,7 & 1,0 \\
\hline Sí & 53 & 66,3 & 27 & 33,7 & $1,5(1,20-2,00)$ \\
\hline \multicolumn{6}{|l|}{ Ruido } \\
\hline No & 43 & 40,6 & 63 & 59,4 & 1,0 \\
\hline Sí & 49 & 76,6 & 15 & 23,4 & $1,9(1,40-2,50)$ \\
\hline \multicolumn{6}{|c|}{ Esfuerzo físico } \\
\hline No & 78 & 50,6 & 76 & 49,4 & 1,0 \\
\hline Sí & 14 & 87,5 & 2 & 12,5 & $1,7(1,20-2,50)$ \\
\hline \multicolumn{6}{|c|}{ Posiciones forzadas } \\
\hline No & 46 & 37,4 & 77 & 62,6 & 1,0 \\
\hline Sí & 46 & 97,9 & 1 & 2,1 & $2,6(2,00-3,40)$ \\
\hline \multicolumn{6}{|c|}{ Trabajo intenso } \\
\hline No & 29 & 31,9 & 62 & 68,1 & 1,0 \\
\hline Sí & 63 & 79,7 & 16 & 20,3 & $2,5(1,90-3,30)$ \\
\hline \multicolumn{6}{|l|}{ Monotonía } \\
\hline No & 51 & 41,5 & 72 & 58,5 & 1,0 \\
\hline Sí & 41 & 87,2 & 6 & 12,8 & $2,1(1,60-2,80)$ \\
\hline \multicolumn{6}{|c|}{ Repetitividad } \\
\hline No & 18 & 24,3 & 56 & 75,7 & 1,0 \\
\hline Sí & 74 & 77,1 & 22 & 22,9 & $3,2(2,30-4,40)$ \\
\hline
\end{tabular}

* Razón de prevalencia; ** Intervalo con 95\% de confianza. Modelos de regresión logística ajustados por edad y por antigüedad en el IMSS. En todos los casos las diferencias fueron significativas.

gencias que pueden ser considerados, en su conjunto, como causantes de estrés laboral y que influyen, de manera importante y directa, en la salud mental de las trabajadoras. Para este estudio, y dado que son causantes de estrés conocidos, se tomaron en cuenta los siguientes: calor, ruido, esfuerzo físico, posiciones forzadas, trabajo intenso, trabajo monótono y trabajo repetitivo.

La morbilidad mental de las trabajadoras expuestas a calor y a ruido intenso durante gran parte o toda la jornada se eleva notablemente en relación con las no expuestas. En ambos casos la diferencia es significativa (Tabla 3). De la misma manera, el esfuerzo físico mostró diferencias muy claras en la morbilidad mental entre las trabajadoras sometidas a él, por un lado, de manera moderada o pesada y, por el otro, las poco o no expuestas. Muchas de las trabajadoras estudiadas tienen que adoptar posiciones forzadas durante gran parte de su jornada laboral. Las más frecuentes son: permanecer de pie mucho tiempo en un mismo sitio y estar encorvada o sentada durante largo tiempo. A 
esto se agrega la falta de pausas, lo que muchas veces hace imposible el cambio de posición. La diferencia es significativa, siendo el riesgo casi tres veces mayor en las expuestas (Tabla 3 ).

El trabajo intenso es otra de las características de este grupo de trabajadoras. Esto se debe en general al ritmo acelerado ocasionado por las múltiples actividades que necesitan efectuar en un tiempo corto. Esto origina que el grado de control que la trabajadora ejerce sobre su actividad disminuya y aumente el grado de atención que tiene que poner en las tareas, lo que incrementa el estrés agudo y crónico y, por lo tanto, la morbilidad mental. Se presenta un riesgo mayor del doble en las expuestas, comparadas con las trabajadoras no sometidas a trabajo intenso. También el trabajo monótono es un causante de estrés, ya que éste provoca aburrimiento y hastío, pero también, cansancio mental y depresión, lo que a su vez se relaciona con fatiga patológica. El riesgo es dos veces mayor en las expuestas. De manera similar, el trabajo repetitivo tiene un efecto negativo. La repetición de una misma tarea, además de generar problemas musculoesqueléticos, provoca hastío, cansancio mental y aburrimiento. En la Tabla 3 se aprecia que el riesgo es tres veces mayor en las expuestas.

Para completar la visión acerca de las causas laborales generadoras de problemas de salud se llevó a cabo un análisis por conglomerados (clusters) con las principales variables que se analizaron, tanto del trabajo doméstico como del trabajo asalariado. A través de este análisis se pudieron diferenciar grupos de trabajadoras que, aunque comparten ciertas condiciones, son distintas en otras. Así, pretendimos ver los efectos en la salud mental de dos grupos de trabajadoras de la salud con diversos grados de exposición a causantes de estrés laboral. En la Figura 1 se aprecia que el grupo sometido simultáneamente a un mayor grado de exposición a calor, ruido, esfuerzo físico, posiciones forzadas, trabajo intenso y trabajo repetitivo presentaba un riesgo 6,5 veces mayor a irritabilidad y 3,6 veces mayor a fatiga que el grupo de trabajadoras con menor grado de exposición. Es interesante observar que ambos grupos presentan condiciones casi idénticas en cuanto a la carga de trabajo doméstica. Así, el conjunto de la carga del trabajo asalariado es, en sí mismo, un generador de enfermedad en estas mujeres, independientemente del trabajo doméstico. Las causas principales de estos padecimientos se encuentran estrechamente relacionadas con un trabajo exigente y descalificado y con exigencias ergonómicas y físicas relacionadas con el tipo de actividad.
En la Figura 2 se muestra también un análisis por conglomerados (clusters), pero ahora con variables del trabajo doméstico y de la valoración que hacen las trabajadoras sobre el trabajo asalariado. Es interesante notar que se aprecia una situación similar a la anterior en cuanto a la carga de trabajo doméstica, ya que ambos grupos comparten las mismas condiciones. Sin embargo, se diferencian claramente en cuanto al contenido del trabajo, expresado a través de las posibilidades que tienen estas mujeres de desarrollar sus capacidades y su creatividad y la satisfacción que perciben sobre sus actividades. Se pudo apreciar que aquel grupo que tenía un casi nulo contenido de trabajo (expresado por su capacidad de desarrollo, satisfacción y creatividad) presentaba muchos mayores problemas, también como en el caso anterior, de irritabilidad y fatiga. En este caso es una relación inversamente proporcional, es decir, a menor contenido del trabajo mayores problemas de salud. Así, el riesgo de irritabilidad fue 15,5 veces mayor en las trabajadoras con actividades con muy bajo contenido que aquéllas que su actividad tiene un alto contenido de trabajo. Lo mismo sucedió con la fatiga que las primeras presentaron un riesgo 5,8 veces mayor. En ambos casos, las diferencias fueron significativas.

\section{Discusión}

Un hallazgo interesante de esta investigación es que el riesgo de padecer algún trastorno mental se incrementa $50,0 \%$ en el caso de las trabajadoras que tienen pareja. Una posible explicación es que las responsabilidades de la mujer trabajadora, en vez de disminuir al tener una pareja, se aumentan, ya que, además de las responsabilidades del hogar y de los hijos, tiene la del compañero. Por otra parte, tener hijos, además de significar una relación afectiva importante, implica una exigencia que puede tener, bajo ciertas circunstancias, una acción estresante de gran magnitud, causante de desequilibrio emocional. Por eso, las trabajadoras con hijos tuvieron un riesgo mucho mayor. De la misma manera sucedió con las mujeres que no contaban con alguna ayuda para llevar a cabo las actividades domésticas.

En síntesis, se muestra cómo algunos aspectos de la vida cotidiana en el ámbito doméstico están en estrecha relación con la generación de trastornos mentales. Esto se refleja, sobre todo, en algunas exigencias relacionadas con actividades fatigosas, tales como, si se tienen hijos y el número de ellos y la falta de ayu- 
Comparación entre dos conglomerados de trabajadoras de la salud con diferentes grados de exposición.

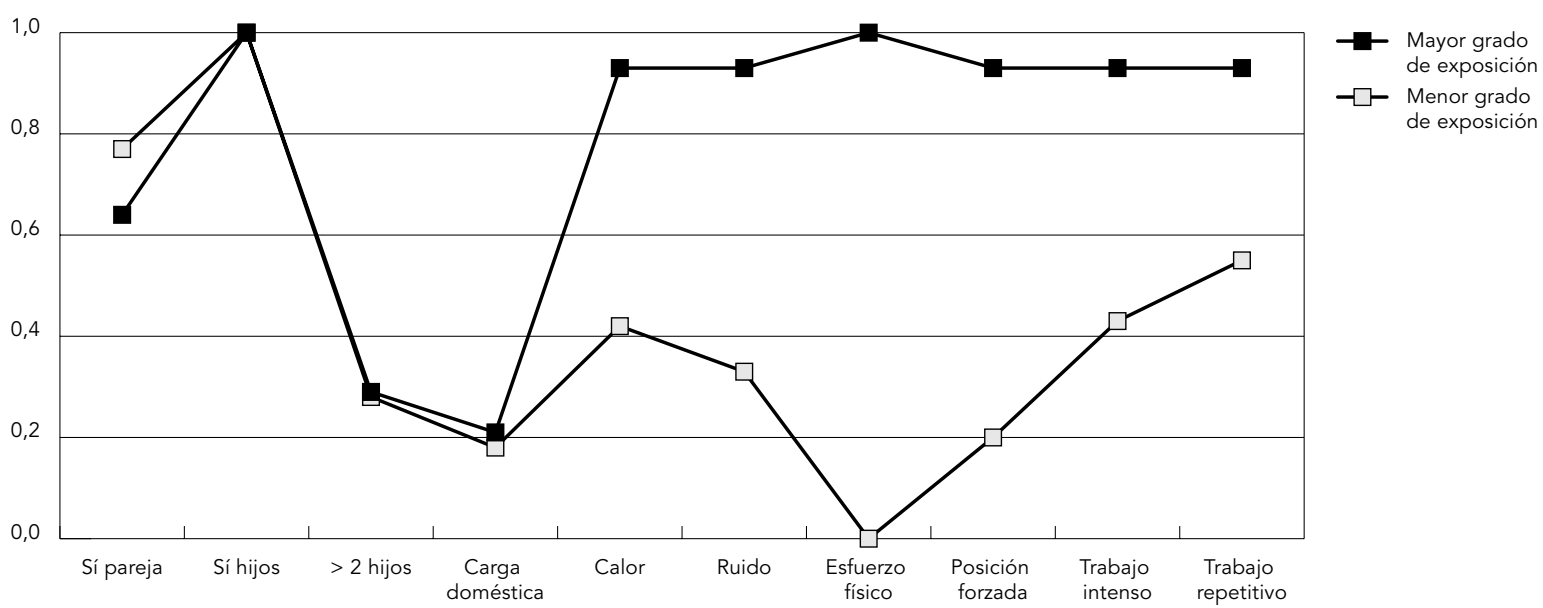

Figura 2

Comparación entre dos conglomerados de trabajadoras de la salud con diferencias en el contenido de trabajo.

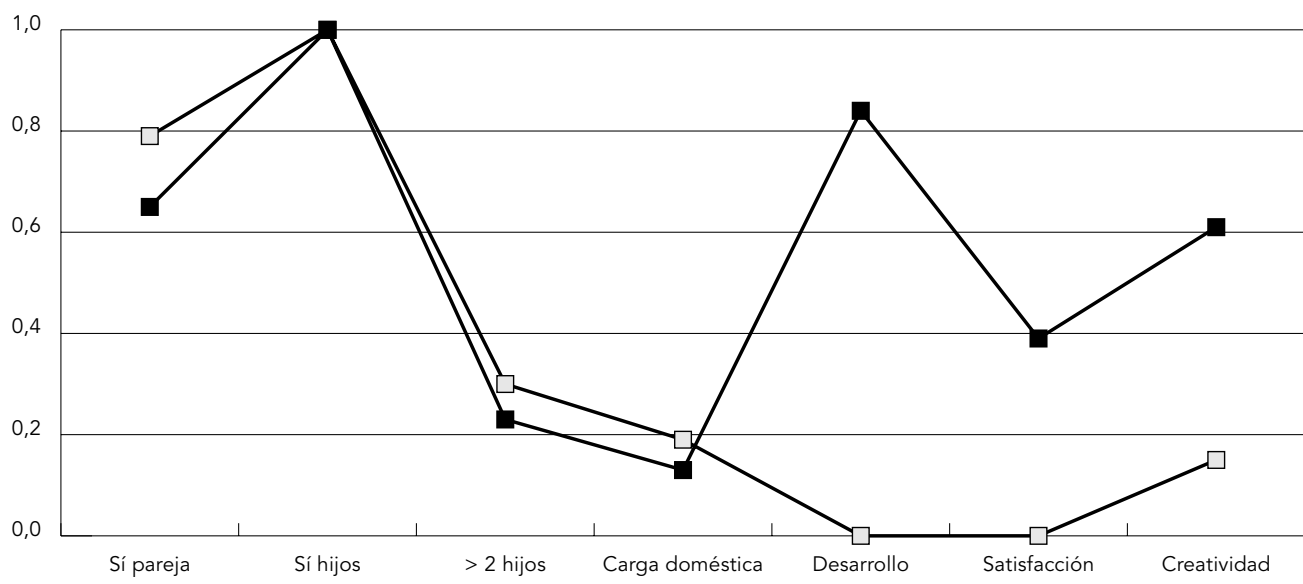

da para las actividades domésticas. Pero también aparece como importante la presencia de la pareja que, al contrario de una situación de justicia y equidad, incrementa el trabajo doméstico de estas mujeres.

Los principales problemas que se encontraron en relación con las actividades remuneradas fueron de varios órdenes: en primer lugar que el tiempo de trabajo es una variable importante en la generación de estrés y de enfermedad mental, no sólo por la extensión de la jornada en sí misma, sino por la forma en que las mujeres trabajadoras tienen que resolver sus actividades extralaborales, sobre todo aquéllas que tienen niños pequeños. Las trabajadoras que laboran jornada completa presentaron mayor morbilidad mental. En segundo lugar, algunas variables relacionadas con el ausentismo, en particular, las incapacidades médicas son un buen indicador en relación con el perfil patológico mental. 
La valoración positiva que la población tiene sobre su trabajo es sustancial para un equilibrio emocional. Si la trabajadora considera que las condiciones son apropiadas favorece de manera importante su salud mental. Por el contrario, si considera inapropiadas esas condiciones de trabajo se incrementa el riesgo de padecer trastornos psíquicos o psicosomáticos e, incluso, la presencia de fatiga. Las trabajadoras que contestaron que sí estaban preocupadas por los daños a la salud que el trabajo les pudiera ocasionar tuvieron una frecuencia de morbilidad mental más elevada. Así, la conciencia sobre la peligrosidad del trabajo que realizan tiene consecuencias en su integridad mental. Es de suponerse que esta situación está estrechamente relacionada con las condiciones objetivas de peligrosidad.

La sensación de bienestar al realizar las actividades asalariadas puede tener un efecto protector para la salud mental, pero también, la ausencia de ella puede tener efectos negativos, como los que encontramos en este estudio. Otras investigaciones también han encontrado situaciones similares 22,23.

Muchos investigadores estudian en la actualidad la relación del control y el contenido del trabajo con los padecimientos mentales 21,26. En esta investigación hubo hallazgos relevantes en este sentido, ya que el desarrollo de habilidades y destrezas en el trabajo, como la satisfacción y la creatividad en el mismo, fueron aspectos "protectores" o preventivos de trastornos mentales y de fatiga. Dicho de otra manera, la carencia de contenido del trabajo es un elemento generador de morbilidad mental.

Las actuales condiciones de trabajo de la población en estudio determina la presencia de un variado e importante número de causantes de estrés laboral, ya que este tipo de trabajadoras tiene que: efectuar varias tareas simultáneamente, subir y bajar escaleras, mantener elevada atención, asumir la responsabilidad de asistir con seguridad al paciente, mantener gran control sobre su trabajo, repetir con frecuencia indicaciones específicas a los usuarios, lo que evidentemente ocasiona que la trabajadora realice un importante esfuerzo físico y se someta a posiciones forzadas durante gran parte de la jornada, además de trabajo intenso, lo que mostró diferencias muy claras en la morbilidad mental entre las trabajadoras expuestas y aquéllas que no lo estaban.

Para completar la visión acerca de las causas generadoras de problemas de salud mental, se llevó a cabo un análisis por conglomerados (clusters) con las principales variables tanto del trabajo doméstico como del trabajo asa- lariado. Fue un hallazgo encontrar que las diferencias entre los conglomerados se daban entre las variables relacionadas con el trabajo asalariado y que las variables relacionadas con la vida fuera del centro de trabajo eran muy similares (casi idénticas) entre los grupos. Esto habla de la semejanza de estos grupos en cuanto a sus condiciones de vida, pero también de que el trabajo asalariado en sí mismo es determinante de una parte muy importante de los padecimientos mentales de este grupo de trabajadoras de la salud y que, además, pueden precisarse aquéllas variables que principalmente determinan cierto tipo de padecimientos mentales. La importancia mayor radica en que, a pesar de lo que se ha dicho de que las causas de la morbilidad mental en las mujeres trabajadoras se debe a una multiplicidad de situaciones, lo cual es cierto, y que son del ámbito asalariado y no asalariado, lo cual también es cierto, es también necesario precisar las causas inmediatas que generan estos trastornos y que, al menos una parte importante de ellas, son específicamente del ámbito del trabajo asalariado, ya que esto permite proponer acciones específicas para enfrentarlas. Este análisis relevó que la valoración que hacen las trabajadoras sobre su trabajo y el contenido del mismo son también determinantes en la aparición de trastornos mentales.

Por último, un aspecto relevante de los hallazgos es que las trabajadoras de la salud de los diferentes servicios y puestos comparten de manera similar riesgos y exigencias y daños a la salud. Dicho de otra manera, se pensaba que, como en otros estudios, el tipo de servicio o el puesto de trabajo serían las variables determinantes que explicaran las diferencias en la morbilidad mental, pero no fue así. Otras variables tuvieron mayor poder explicativo en la generación de los trastornos psíquicos, psicosomáticos y de la fatiga.

\section{Conclusiones}

Esta investigación pretendió entender la relación entre elementos que son muy relevantes en nuestro campo de estudio: la situación de la mujer y, en particular, de las trabajadoras de la salud; las condiciones de la vida cotidiana en el ámbito doméstico; las condiciones del trabajo remunerado y la percepción que tienen estas mujeres sobre ellas; los riesgos y exigencias a los que están principalmente expuestas y, por último, algunos de los trastornos mentales íntimamente relacionados con las situaciones anteriores. 
Los hallazgos mostraron, en primer lugar, algo que con frecuencia se menciona, pero que es poco reconocido: que los trastornos mentales están relacionados con todos los ámbitos de la vida de las mujeres. Sin embargo, este aspecto multigenerador no hace que la producción de estos problemas sea caótica o que no puedan estudiarse con rigor las causas que los originan. Esto fue quizá el elemento central del trabajo, ya que se encontraron algunas causas similares a las de otras investigaciones, pero también se obtuvieron explicaciones distintas a las ya conocidas, en la generación de trastornos psíquicos y psicosomáticos.

En relación con lo que llamamos la vida cotidiana en el ámbito doméstico se encontró que las mujeres que son madres reportaron una morbilidad mayor. La explicación probablemente se deba a que tienen un mayor desgaste físico y emocional ocasionado por el trabajo y la responsabilidad que representa el cuidado de los hijos. Así, también se evidenció en la investigación que a mayor número de hijos mayor frecuencia de trastornos mentales. De la misma manera sucedió en el caso de las mujeres que no contaban con ayuda para el trabajo doméstico. Esta situación, como la anterior, trae consigo un gran desgaste físico y mental y diferencia, claramente, a unas mujeres de otras. Así, las que no tienen ayuda necesitan realizar una nueva jornada de trabajo, inmediatamente después de terminar con las actividades que les exige el trabajo asalariado. Las otras, aunque requieren realizar algunas actividades, éstas no implican un estrés tan grande ni un esfuerzo físico y mental de la misma magnitud. Un aspecto importantísimo de considerar es que las mujeres con pareja presentan mucha mayor morbilidad mental, lo que hace necesario profundizar en este aspecto, ya que aquí sólo se esbozaron algunas posibles explicaciones.

En relación con las características del trabajo asalariado, los hallazgos mostraron que el área y puesto en este tipo de trabajo no son los principales determinantes en las diferencias en la morbilidad mental como otros aspectos laborales. Sin embargo, hubo resultados muy claros en relación con el control y el contenido del trabajo, ya que la falta de desarrollo de habilidades y de destrezas en el trabajo, así como la insatisfacción y la falta de creatividad en el mismo fueron aspectos generadores de trastornos mentales y de fatiga o, dicho de otra manera, el mayor control y el mayor contenido del trabajo fueron elementos "protectores" o preventivos de estos trastornos. Una de las principales formas en que se puede contrarrestar el efecto de los causantes de estrés es cuando el trabajador puede ejercer control sobre la ocurrencia de los eventos externos. Hay "control" cuando el individuo puede ejercer determinada influencia en la ocurrencia de las respuestas 27. En situaciones de falta de control el individuo experimenta ciertas reacciones comunes como la ansiedad y el miedo y si además se prolongan dichas situaciones, los efectos serán más graves y pueden sobrevenir trastornos como los psíquicos y los psicosomáticos. Sin embargo, no sólo el control individual y colectivo es importante para evitar o reducir el estrés y estos trastornos. La modificación de los métodos de trabajo y la adecuada formación y capacitación permanente 28 , junto con la supresión de ciertas exigencias laborales son elementos imprescindibles en el combate contra las condiciones nocivas de trabajo y de salud.

\section{Resumen}

Se estudió la relación entre condiciones de vida, trabajo y trastornos mentales en trabajadoras de la salud del Instituto Mexicano del Seguro Social (IMSS). La población se integró por médicas, enfermeras, personal de laboratorio y asistentes médicas $(n=170)$. La información principal se obtuvo de una encuesta, validada en población trabajadora mexicana. Se encontró que los trastornos mentales están relacionados con todos los ámbitos de la vida de las mujeres. En relación con el ámbito doméstico, se muestra que las mujeres con mayor morbilidad mental son: las que son madres; las que tienen un mayor número de hijos; aquéllas que no cuentan con ayuda para el trabajo doméstico; y las que tienen pareja. En relación con el trabajo asalariado, la morbilidad mental se encontró asociada con la duración de la jornada, el ausentismo y la falta de contenido del trabajo. El desarrollo de habilidades, la satisfacción y la creatividad fueron aspectos "protectores" o preventivos de trastornos mentales y de fatiga. Los principales riesgos y exigencias que actuaron como causantes de estrés fueron: el calor, el ruido, el esfuerzo físico, las posiciones forzadas y el trabajo intenso y repetitivo.

Condiciones de Trabajo; Condiciones de Vida; Trastornos Mentales; Fatiga 


\section{Colaboradores}

M. Noriega participó de la elaboración del proyecto y de la metodología, dirección de la investigación, búsqueda y lectura de las principales fuentes bibliográficas consultadas, convenio con la institución de salud donde se hizo la investigación; procesamiento y análisis de los datos, y elaboración del artículo. G. Gutiérrez se responsabilizó de la búsqueda inicial de bibliogra- fía, elaboración de fichas bibliohemerográficas; fue la principal colaboradora en el trabajo de campo, captura de la información y procesamiento incipiente de la misma. I. Méndez fue el principal colaborador en el análisis estadístico, lectura de los materiales que se generaban. M. Pulido participó en la lectura y elaboración de los materiales bibliográficos definitivos.

\section{Referencias}

1. Heller A. Sociología de la vida cotidiana. 5a Ed Barcelona: Ediciones Península; 1998.

2. Lennon MC, Rosenfield S. Women and mental health: the interaction of job and family conditions. J Health Soc Behav 1992; 33:316-27.

3. Vite S. Antigüedad laboral y su relación con la autoestima de la mujer. Revista Mexicana de Psicología 1990; 7:51-5.

4. Hall EM. Gender, work control, and stress: a theoretical discussion and an empirical test. Int J Health Serv 1989; 19:725-45.

5. Phelps S. When women lose their jobs. Pers J 1991; 70:64-9.

6. Trucco M, Valenzuela P, Trucco D. Estrés ocupacional en personal de salud. Rev Med Chil 1999; 127:1453-61.

7. Yassi A. Centros y servicios de asistencia sanitaria. In: Stellman JM, editor. Enciclopedia de salud y seguridad en el trabajo. 3a Ed. v. 3. Madrid: Organización Internacional del Trabajo/Ministerio de Trabajo y Asuntos Sociales; 2001. p. 97.2-13.

8. Kelloway EK, Barlin J. Job characteristics, role stress and mental health. Journal of Occupational Psychology 1991; 64:291-304.

9. Ramirez AJ, Graham J, Richards MA, Cull A, Gregory WM. Mental health of hospital consultants: the effects of stress and satisfaction at work. Lancet 1996; 347:724-8.

10. Acevedo D. Género y flexibilización del trabajo: impacto en la salud. Cuadernos Mujer Salud 1997; 2:28-36

11. Díaz X, Mendel J. Mujer, trabajo y salud: los daños ocultos. Cuadernos Mujer Salud 1997; 2:37-46.

12. Waldron I, Weiss CC, Hughes ME. Interacting effects of multiples roles on women's health. J Health Soc Behav 1998; 39:216-36.

13. de Jonge J, Mulder MJ, Nijhuis FJ. The incorporation of different demand concepts in the job demand-control model: effects on health care professionals. Soc Sci Med 1999; 48:1149-60.

14. Blane D, Berney L, Montgomery SM. Domestic labour, paid employment and women's health: analysis of life course data. Soc Sci Med 2001; 52:959-65.

15. Leppänen RA, Olkinuora MA. Psychological stress experienced by health care personnel. Scand J Work Environ Health 1987; 13:1-8.

16. Kawakami N, Araki S, Kawashima M. Effects of job stress on ocurrence of mayor depression in
Japanese industry: a case-control study nested in a cohort study. J Occup Med 1990; 32:722-5.

17. Estryn-Béhar M, Kaminski M, Peigne E, Bonnet N, Vaichere E, Gozlan C, et al. Stress at work and mental health status among female hospital workers. Br J Ind Med 1990; 47:20-8.

18. Hardy GE, Shapiro DA, Borrill CS. Fatigue in the workforce of national health service trusts: levels of symptomatology and links with minor psychiatric disorder, demographic, occupational and work role factors. J Psychosom Res 1997; 43:83-92.

19. American Psychiatric Association. Manual diagnóstico y estadístico de los trastornos mentales: DSM-III-R. Barcelona: Editora Masson; 1988.

20. American Psychiatric Association. Manual diagnóstico y estadístico de los trastornos mentales: DSM-IV. Barcelona: Editora Masson; 1995.

21. Karasek R, Theorell T. Healthy work: stress, productivity, and the reconstruction of working life. New York: Basic Books; 1990.

22. Thomsen S, Soares J, Nolan P, Dallender J, Arnetz B. Feelings of profesional fulfilment and exhaustion in mental health personnel: the importance of organizational and individual factors. Psychoter Psychosom 1999; 68:157-64.

23. Aasland OG, Olff M, Falkum E, Schweder T, Ursin H. Health complaints and job stress in Norwegian physicians: the use of an overlapping questionnaire design. Soc Sci Med 1997; 45:1615-29.

24. Bourbonnais R, Comeau M, Vézina M, Dion G. Job strain, psychological distress and burnout in nurses. Am J Ind Med 1998; 34:20-8.

25. Felton JS. Burnout as a clinical entity - its importance in health care workers. Occup Med (Lond) 1998; 48:237-50.

26. Frankenhaeuser M. A biopsychosocial approach to work life issues. Int J Health Serv 1989; 19:74758.

27. Aronsson G. Dimensions of control as related to work organization, stress and health. Int J Health Serv 1989; 19:459-68.

28. Rossi K, Heinonen K, Heikkinen MR. Factors affecting the work of an occupational health nurse. Occup Med (Lond) 2000; 50:369-72.

Recibido el 12/Sep/2003

Versión final presentada el 06/May/2004

Aprobado el 26/May/2004 